1 Centro Universitário

Tabosa de Almeida

(Asces/Unita) - Caruaru (PE), Brasil. Universidade de Pernambuco (UPE), Faculdade de Ciências Médicas (FCM) - Recife (PE), Brasil.

eudes/orena@hotmail.com

2 Centro Universitário Tabosa de Almeida

(Asces/Unita) - Caruaru (PE), Brasil.

joaolxnetto@gmail.com

${ }^{3}$ Centro Universitário

Tabosa de Almeida

(Asces/Unita) - Caruaru

(PE), Brasil.

victor.fhn@gmail.com

4 Universidade Federal de Pernambuco (UFPE) Recife (PE), Brasil.

petroniocarla@uol.com.br

5 Faculdade Pernambucana de Saúde (FPS) - Recife (PE), Brasil.

suelem.barros@yahoo.

com.br

\section{Análise das iniciativas do Poder Legislativo estadual de Pernambuco para o setor saúde}

\author{
Analysis of the initiatives of the Pernambuco state Legislative Power \\ for the health sector
}

José Eudes de Lorena Sobrinho', João Inácio Júnior Neto², Victor Hugo de França do

Nascimento ${ }^{3}$, Petrônio José de Lima Martelli4, Suélem Barros de Lorena ${ }^{\mathbf{5}}$

DOI: 10.1590/0103-1104201811711

RESUMO O objetivo desta pesquisa foi analisar as iniciativas do Poder Legislativo estadual de Pernambuco para o setor saúde, caracterizando as indicações, requerimentos e projetos de lei quanto às subáreas da saúde e populações beneficiadas. Trata-se de um estudo descritivo e exploratório que utilizou a metodologia qualitativa. Foram utilizadas atas das reuniões ocorridas no ano de 2015 pela Assembleia Legislativa do Estado de Pernambuco, disponibilizadas no portal on-line. Das 117 proposições, 81 foram específicas da saúde, sendo a maioria conduzida por parlamentares da base aliada ao Executivo. Temas como alimentação/água, saúde do trabalhador e serviços e ações de saúde foram mais frequentes.

PALAVRAS-CHAVE Política social. Poder Legislativo. Saúde.

ABSTRACT The objective of this research is to analyze the initiatives of the state Legislative Power of Pernambuco for the health sector, characterizing the indications, requirements and bills of law regarding the health subareas and beneficiary populations. It was a descriptive and exploratory study that used the qualitative methodology. Minutes of the meetings held in the year 2015 by the Legislative Assembly of the State of Pernambuco, made available online, have been used. Of the 117 proposals, 81 were health-specific, with the majority being led by parliamentarians from the base allied to the Executive. Issues such as food/water, occupational health, and health services and actions were more frequent.

KEYWORDS Public policy. Legislative. Health. 


\section{Introdução}

Há uma diversidade conceitual disponível na literatura sobre políticas públicas formulada por múltiplos autores, que tratam do 'conteúdo concreto e simbólico das decisões políticas e do seu processo de construção e atuação', com o protagonismo de atores governamentais na sua formulação 1 .

Para definir adequadamente as políticas públicas, três elementos são fundamentais: o reconhecimento do protagonismo do governo, a importância da decisão fundamental pelo governo e o fato de que essa decisão carrega uma intencionalidade por parte dos agentes do governo ${ }^{2}$.

Para uma melhor compreensão da complexidade envolvida na política pública, um dos modelos teóricos elaborados é denominado 'ciclo da política', o qual está organizado em sete fases: identificação do problema, formação da agenda, formulação de alternativas, tomada de decisão, implementação, avaliação e extinção'.

Ganhará destaque neste trabalho a fase denominada 'formulação de alternativas', considerando que esta é uma das atribuições do Poder Legislativo ${ }^{3}$. Nessa fase, há propostas de soluções para os problemas reconhecidos no primeiro momento, definindo as opções políticas formuladas dentro do governo. Deve-se levar em consideração os limites de cada solução proposta, as restrições de recursos, o apoio político, bem como as restrições legais. Ressalte-se que grupos de interesse podem tomar posição sobre as questões, apoiando-as ou não - ao subsidiar campanhas, por exemplo -, o que influenciaria a formulação da política ${ }^{1}$.

Os atores participantes desse processo podem ser diferenciados em 'participantes visíveis' e 'participantes invisíveis'. Os visíveis são os que participam diretamente do processo político, como os representantes do governo (políticos eleitos), assim como a mídia e os partidos políticos. Por sua vez, os atores invisíveis são os acadêmicos, burocratas de carreira e demais participantes que não fazem parte do governo e que teriam uma importância secundária na definição da agenda, porém com maior poder de influência da escolha das alternativas ${ }^{4}$.

Os deputados estaduais, enquanto políticos eleitos, constituem-se como 'policy makers', ou seja, formuladores das políticas, no grupo dos 'atores visíveis's. Os formuladores das políticas que ocupam cargos públicos em mandatos eletivos são denominados 'atores políticos'; e é sobre esta categoria que o estudo em tela irá se debruçar6.

Estudos que abordam a temática do Poder Legislativo e sua atuação na área da saúde têm focado no âmbito federal ao analisar as atividades do Congresso Nacional e o impacto das emendas parlamentares no orçamento da União ${ }^{7-9}$. Ainda na esfera do Poder Legislativo Federal, foram desenvolvidos estudos que trataram de analisar iniciativas voltadas a políticas setoriais de saúde, como as de saúde mental, as de combate às drogas ${ }^{10} \mathrm{e}$ as de saúde bucal ${ }^{11 .}$.

Estudos na linha do controle social e atividade legislativa merecem destaque na medida em que esse princípio, quando bem exercido, constitui-se como importante mecanismo de prevenção da corrupção e de fortalecimento da cidadania, necessidades emergentes na atualidade do País ${ }^{12}$.

Uma lacuna nesse campo de análise é observada no âmbito dos poderes legislativos dos estados e municípios, motivando o desenvolvimento deste estudo na dimensão do estado de Pernambuco.

Dessa forma, o objetivo desta pesquisa é analisar as iniciativas do Poder Legislativo estadual de Pernambuco para o setor saúde, caracterizando as indicações, proposições, requerimentos e projetos de lei quanto às subáreas da saúde favorecidas e populações beneficiadas, verificando a atuação da Comissão da Saúde do Poder Legislativo estadual de Pernambuco.

\section{Material e métodos}

Tratou-se de um estudo analítico e exploratório que utilizou a metodologia qualitativa. Foram utilizados dados secundários 
provenientes das atas das reuniões ocorridas no ano de 2015 pela Assembleia Legislativa do Estado de Pernambuco (Alepe), disponibilizadas para acesso público no portal on-line dessa instituição. Foram excluídas deste estudo as atas provenientes das reuniões solenes ocorridas na Alepe, por se constituírem como atos comemorativos e sem perspectiva de discussão técnica; e também aquelas provenientes de período anterior ou posterior ao recorte temporal estabelecido (2015).

Os procedimentos analíticos utilizados por esta pesquisa foram aqueles recomendados por Bardin: pré-análise, análise do material e tratamento dos resultados ${ }^{13}$. A primeira etapa consistiu em realizar uma leitura superficial do material para conhecer a estrutura e extrair as impressões iniciais; em seguida, procedeu-se à análise propriamente dita, na qual os dados foram codificados e categorizados, conferindo tratamento aos resultados encontrados ${ }^{\mathbf{1 4}}$.

As principais vantagens apontadas por Gil com o uso das fontes documentais está em possibilitar o conhecimento do passado, investigar os processos de mudança social e cultural, menor custo e favorecer a obtenção de dados sem o constrangimento dos sujeitos ${ }^{15}$.

Em conformidade com a Resolução $\mathrm{n}^{\mathrm{o}}$ 466/2012 do Conselho Nacional de Saúde, esta pesquisa dispensou apreciação pelo Comitê de Ética em Pesquisa por não envolver diretamente seres humanos.

\section{Resultados e discussão}

O estudo trouxe a possibilidade de traçar um observatório da atividade parlamentar em Pernambuco no âmbito da saúde em 2015, onde a Comissão de Assistência Social e Saúde recebeu um total de 117 proposições, sendo 81 específicas para a área da saúde. Destas proposições, 80 foram oriundas de membros do Legislativo estadual, e apenas 1 pelo Poder Executivo estadual, evidenciando uma discrepância de cenário quando em comparação com o âmbito federal, em que o Executivo se constitui como principal agente de políticas para a área da saúde. No período de 2003 a 2006, o Executivo federal foi autor de 129 proposições; e o Legislativo, apenas $25^{8}$. Este fato demonstra um baixo protagonismo do Executivo estadual na formulação de políticas de saúde, concentrando sua atuação na execução de políticas anteriormente formuladas e implementadas.

Observa-se ainda, no panorama estadual, uma grande pluralidade de partidos políticos que apresentaram proposições para o setor saúde (conforme tabela 1), sendo, ao todo, 13 partidos, incluindo a mesa diretora da Comissão de Saúde e Assistência Social, que também apresentou ação nesse sentido.

Tabela 1. Caracterização dos sujeitos do estudo de acordo com os 7 segmentos

\begin{tabular}{lrr}
\hline Partidos políticos & Quantidade de Parlamentares & Total de proposições \\
\hline PSB - Partido Socialista Brasileiro & $7^{\star}$ & $9^{*}$ \\
PP - Partido Progressista & 4 & 13 \\
PTB - Partido Trabalhista Brasileiro & 3 & 13 \\
PRB - Partido Republicano Brasileiro & 3 & 6 \\
PMDB - Partido do Movimento Democrático Brasileiro & 2 & 7 \\
PSDB - Partido da Social Democracia Brasileira & 2 & 2 \\
PSL - Partido Social Liberal & 2 & 13 \\
PR - Partido da República & 2 & 7 \\
\hline
\end{tabular}




\begin{tabular}{|c|c|c|}
\hline PSD - Partido Social Democrático & 2 & 2 \\
\hline PT - Partido dos Trabalhadores & 1 & 3 \\
\hline PDT - Partido Democrático Trabalhista & 1 & 1 \\
\hline PCdoB - Partido Comunista do Brasil & 1 & 2 \\
\hline PSOL - Partido Socialismo e Liberdade & 1 & 2 \\
\hline Mesa Diretora & 1 & 1 \\
\hline TOTAL & 32 & 81 \\
\hline
\end{tabular}

Essa pluralidade, porém, não representa diretamente o nível de confiança dos cidadãos nas suas instituições representativas, principalmente nos partidos políticos, sendo esse índice no Brasil demasiadamente baixo, principalmente quando comparado a países que possuem regimes democráticos estabelecidos, inclusive na América Latina. Isso se reflete em uma falta de legitimidade, fator imprescindível para que os parlamentares sejam capazes de exercer de forma plena suas funções, fazendo com que a população não tenha suas demandas atendidas, gerando, por sua vez, um descrédito ainda maior nos representantes escolhidos, o que acaba por se tornar uma reação em cadeia de difícil solução ${ }^{16,17}$.

Outro fator de importância para uma melhor análise da situação é a quantidade de parlamentares por partido em relação à quantidade de proposições, sendo possível observar que o fato de haver partidos com uma quantidade superior de parlamentares não implica uma relação direta com o número de proposições ou ação deles no quesito saúde, havendo partidos de menor expressão apresentando número inferior de parlamentares, e sendo tão ou até mesmo mais ativos para o setor.

Em Pernambuco, de um total de 31 parlamentares que realizaram propostas para a saúde, apenas 8 foram de partidos que não integram a base aliada do Poder Executivo estadual de Pernambuco (PTB, PRB, PT e PSOL). Das 81 propostas, 24 foram provenientes dos partidos de oposição.

Em relação a Pernambuco, os parlamentares que mais propuseram sobre o tema saúde foram empresários, administradores e economistas (gráfico 1), em contraposição à realidade nacional, em que, na maioria das vezes, os parlamentares mais atuantes no quesito saúde possuem formação nessa área ${ }^{7,8}$.

Argumenta-se sobre esse item, que há grande influência do interesse privado sobre a saúde pública, em especial das empresas de planos de saúde, por terem sido financiadoras diretas de um grande número de deputados estaduais, federais e governadores, de partidos e correntes ideológicas distintas em uma análise nacional ${ }^{18}$. Especificamente para o estado de Pernambuco, esta análise não foi realizada. 
Gráfico 1. Perfil profissional dos parlamentares pernambucanos com proposições na área da saúde (2015)

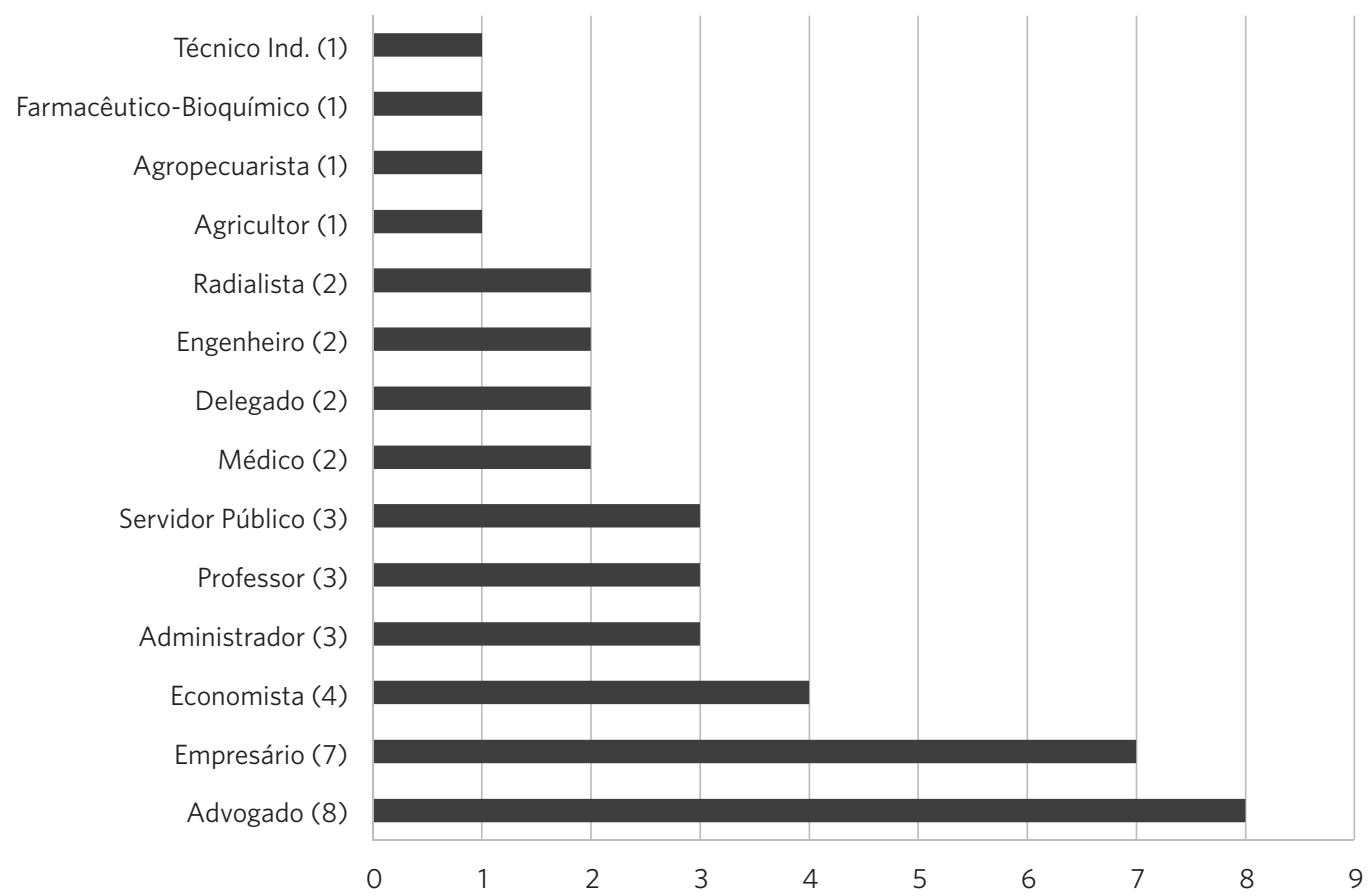

Nota: Alguns parlamentares possuem mais de uma profissão.

Quanto aos temas abordados na Comissão de Saúde e Assistência Social da Alepe no ano de 2015, destacam-se: alimentação/ água, saúde do trabalhador, serviços e ações em saúde e diabetes/hipertensão, conforme tabela 2.

Tabela 2. Temas abordados na Comissão de Saúde e Assistência Social da Assembleia Legislativa do estado de Pernambuco e o quantitativo de proposições, 2015

\begin{tabular}{|c|c|c|}
\hline Assunto & Proposições & Total \\
\hline Alimentação e Água & $\begin{array}{r}№ \text { 99/2015, № 115/2015, № 116/2015, № 141/2015, № 152/2015, № 189/2015, } \\
\text { № 248/2015, № 251/2015, № 362/2015, № 432/2015, № 621/2015 }\end{array}$ & 11 \\
\hline Saúde do Trabalhador & $\begin{array}{r}\text { No 53/2015, № } 107 / 2015 \text {, № 313/2015, № 319/2015, № 364/2015(Radiologia), } \\
\text { № } 374 / 2015 \text {, }\end{array}$ & 6 \\
\hline Serviços e Ações em Saúde & $\begin{array}{r}\text { № 160/2015, № 215/2015, № 369/2015, № 395/2015, № 450/2015, № } \\
\text { 492/2015 }\end{array}$ & 6 \\
\hline Diabetes/Hipertensão & № 17/2015, № 225/2015, № 273/2015, № 380/2015, № 574/2015 & 5 \\
\hline Informações de Saúde & № 246/2015, № 244/2015, № 536/2015, № 545/2015, № 616/2015 & 5 \\
\hline $\begin{array}{l}\text { Eventos/Propagandas - } \\
\text { Saúde }\end{array}$ & № 89/2015, № 366/2015, № 389/2015, № 391/2015, № 442/2015 & 5 \\
\hline Deficiências & № 250/2015, № 332/2015, № 587/2015, № 614/2015 & 4 \\
\hline Medicamentos & № 312/2015, № 463/2015, № 480/2015, № 596/2015 & 4 \\
\hline Câncer & № 125/2015, № 367/2015, № 539/2015 & 3 \\
\hline Primeiros Socorros & № 490/2015, №537/2015, № 606/2015 & 3 \\
\hline
\end{tabular}




\begin{tabular}{|c|c|}
\hline Doenças Renais Crônicas & № 392/2015, № 532/2015 \\
\hline Obesidade & № 263/2015, № 582/2015 \\
\hline Síndrome de Down & № 393/2015, № 579/2015 \\
\hline Parto Humanizado & № 411/2015, № 622/2015 \\
\hline Resíduos & № 247/2015, 261/2015 \\
\hline Visão/Audição & № 311/2015, № 584/2015 \\
\hline Saúde e higienização no Meio Ambiente & № 226/2015, № 601/2015 \\
\hline Doenças Raras & № 153/2015, № 600/2015 \\
\hline Depressão & № 61/2015, № 63/2015 \\
\hline Transtorno de Espectro Autista & № 47/2015, № 240/2015 \\
\hline Violência & № 303/2015 \\
\hline Dependentes de Drogas & № 170/2015 \\
\hline Doadores (sangue/ medula óssea/ órgãos) & № $148 / 2015$ \\
\hline Estudos em Saúde & № 119/2015 \\
\hline Atendimentos & № 60/2015 \\
\hline Saúde Mental & № $466 / 2015$ \\
\hline Epidemias & № $243 / 2015$ \\
\hline Glaucoma & № $235 / 2015$ \\
\hline Aleitamento Materno & № 242/2015 \\
\hline
\end{tabular}

Destacam-se, como assuntos tratados na categoria alimentação/água, os projetos que previam medidas para fiscalização e autuação de estabelecimentos flagrados comercializando produtos vencidos e/ou em más condições de armazenamento (Projeto 99/2015) e o que estabelece a obrigatoriedade de indicação expressa sobre o uso de agrotóxicos nos produtos alimentares comercializados no estado de Pernambuco (Projeto 116/2015). Na categoria saúde do trabalhador, considera-se de relevância o tema de proteção radiológica em estabelecimentos públicos e privados no estado (Projeto 364/2015).

Alguns temas estão em concordância com as necessidades de saúde da população, a exemplo das proposições referentes às doenças do aparelho circulatório, tendo em vista que estas se constituíram 29,59\% das causas de óbitos em Pernambuco no ano de $2010^{19}$. Sobre esse tópico, o Projeto $17 / 2015$ previa prioridade no atendimento aos pacientes portadores de diabetes na rede estadual de saúde, ainda definindo que tais estabelecimentos deveriam fornecer aos pacientes diagnosticados noções básicas sobre os cuidados no tratamento antes de encaminhamento ao especialista. O Projeto 225/2015 definia o mês de setembro como o de prevenção à hipertensão e ao diabetes, denominando-o de 'setembro vermelho'.

No entanto, matérias de grande relevância, como políticas de assistência a neoplasias, doenças do aparelho respiratório e doenças endócrinas que, juntas, corresponderam a 30,07\% das causas de mortalidade no estado também em 2010, não foram contempladas pelas proposições. Também não ocupou posição de destaque o elevado 
número de acidentes envolvendo transportes, fator predisponente de grande oneração na assistência à saúde, posto que, em 2010, o estado apresentou 1.991 óbitos relacionados com esse tipo de causa externa ${ }^{19}$.

\section{Considerações finais}

A atuação do Poder Legislativo estadual é fundamental para a garantia de políticas de saúde que atendam às necessidades da população. O panorama estudado demonstra que temas como alimentação/água, saúde do trabalhador e serviços e ações de saúde foram os mais frequentes nas proposições realizadas para o setor saúde, seguidas de outras voltadas para doenças crônicas não transmissíveis, o que, em parte, corrobora o perfil epidemiológico do estado de Pernambuco. No entanto, agravos como a violência, doenças respiratórias, neoplasias, acidentes de transporte e consumo de drogas, apesar de serem de grande magnitude, não ocuparam o devido espaço na agenda de prioridades.

A maior parte das propostas foi proveniente da base aliada do Poder Executivo estadual, embora sem relação direta com o quantitativo de parlamentares por partido político, sugerindo que a abordagem de temas relativos à saúde não está diretamente ligada à natureza ideológica do partido ao qual está vinculada.

Os dados encontrados contribuem também para a transparência pública e para o controle social em saúde, temas emergentes em tempos da crise política e administrativa que afeta o Brasil.

\section{Referências}

1. Secchi L. Políticas públicas: conceitos, esquemas de análise, casos práticos. 2. ed. São Paulo: Cencage; 2013.

2. Howlett M, Ramesh M, Perl A. Políticas públicas: seus ciclos e subsistemas: uma abordagem integradora. Rio de Janeiro: Elsevier; 2013.

3. Pernambuco. Constituição do Estado de Pernambuco. Recife: Senado; 1989.

4. Kingdon KW. Agendas, Alternatives and Public Policies. New York: Longman; 1994.
5. Serafim MP, Dias RB. Análise de políticas: uma revisão da literatura. Cad Gest Social [internet]. 2012 jan-jun [acesso em 2018 abr 12]; 3(1):121-134. Disponível em: http://www.periodicos.adm.ufba.br/index.php/cgs/article/viewArticle/213.

6. Viana ALA, Baptista TWTF. Análise de políticas de saúde. In: Giovanella L, Escorel S, Lobato LVC, et al, organizadores. Políticas e sistemas de saúde no Brasil. Rio de Janeiro: Fiocruz; 2008. p. 65-105.

7. Baptista TWF, Machado CV, Lima LD, et al. As emendas parlamentares no orçamento federal da saúde. Cad. Saúde Pública [internet]. 2012 dez 
[acesso em 2018 abr 12]; 28(12):2267-2279. Disponível em: http://www.scielo.br/scielo.php?script=sci arttext\&pid=S0102-311X2012001400006\&lng=pt\&t lng=pt.

8. Baptista TWF. Análise da produção legislativa em saúde no Congresso Nacional brasileiro (19902006). Cad. Saúde Pública [internet]. 2010 jan [acesso em 2018 abr 12]; 26(1):97-109. Disponível em: http://www.scielo.br/scielo.php?pid=S0102$-311 X 2010000100011 \&$ script=sci_abstract $\&$ tlng=pt.

9. Pelaez EJA. A frente parlamentar da saúde: temas em pauta e grupos de interesses presentes [trabalho de conclusão de curso]. Rio de Janeiro: Fundação Oswaldo Cruz; 2009.

10. Silva MB, Delduque MC. Patologização e penalização do uso de drogas: uma análise socioantropológica de proposições legislativas (20072010). Physis [internet]. 2015 jan-mar [acesso em 2018 abr 12]; 25(1):231-250. Disponível em: http://www.scielo.br/scielo.php?pid=S0103$-73312015000100231 \&$ script=sci_abstract\&tlng=pt.

11. Penha N, Senda C, Corvino M, et al. Poder Legislativo, leis e saúde bucal dos brasileiros. Rev. Odontol. Univ. Cid. São Paulo [internet]. 2011 set-dez [acesso em 2018 abr 12]; 23(3):245-252. Disponível em: http://publicacoes.unicid.edu.br/index.php/revistadaodontologia/article/download/384/279.

12. Brasil. Controladoria Geral da União. Controle Social: Orientações aos cidadãos para participação na gestão pública e exercício do controle social. Brasília, DF: Controladoria Geral da União; 2012.

13. Bardin L. Análise de conteúdo. Lisboa: Edições 70; 2009.
14. Richardson RJ. Pesquisa social: métodos e técnicas. 3. ed. São Paulo: Atlas; 2011.

15. Gil AC. Métodos e técnicas de pesquisa social. 6. ed. São Paulo: Atlas; 2011.

16. Moisés JA, organizador. O Congresso Nacional, os partidos políticos e o sistema de integridade: representação, participação e controle interinstitucional no Brasil contemporâneo. Rio de Janeiro: Konrad Adenauer Stiftung; 2014.

17. Moisés JÁ, Carneiro GP. Democracia, Desconfiança Política e Insatisfação com o Regime - o Caso do Brasil. Opin Pública [internet]. 2018 jun [acesso em 2018 abr 12]; 14(1):1-42. Disponível em: http:// www.scielo.br/scielo.php?script=sci_arttext\&pid =S0104-62762008000100001.

18. Schefer M, Bahia L. Representação política e interesses particulares na saúde: o caso do financiamento de campanhas eleitorais pelas empresas de planos de saúde privados no Brasil. Interface [internet]. 2011 jul-set [acesso em 2018 abr 12]; 15(38):947-956. Disponível em: http://www. scielo.br/scielo.php?script=sci_arttext\&pid=S1414$-32832011000300030 \& \operatorname{lng}=$ en\&nrm=iso\&tlng=pt.

19. Pernambuco. Governo do Estado. Secretaria Estadual de Saúde. Plano Estadual de Saúde 2012-2015. Recife: Governo do Estado; 2012.

Recebido em 22/10/2017

Aprovado em 09/05/2018

Conflito de interesses: inexistente

Suporte financeiro: não houve 